\title{
Optimal Design for Matching Network of L-band Low Noise Amplifier
}

\author{
Zhang Yisong ${ }^{1, a}$, Lin Fumin ${ }^{2, b}$ and Zeng Liuxing ${ }^{3, c}$ \\ ${ }^{1,2,3}$ School of Physics and Optoelectronics Engineering, Guangdong University of technology, \\ Guangzhou, 510006, PR China

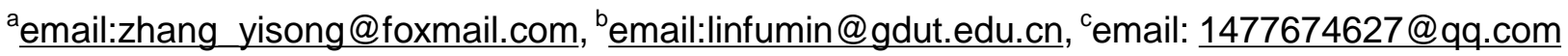

Keywords: lumped elements; microstrip line; matching network; impedance matching

Abstract. This paper introduces a matching network composed of lumped elements and transmission lines, which is more accurate and compact compared to the traditional one. Meanwhile, the added microstrip lines is used for bonding pads and connection lines on printed circuit board (PCB), and the dimensions of these microstrip lines can be adjusted to compensate for the errors brought by the real elements without changing any values of placed inductors or capacitors. This method makes the design of low noise amplifier (LNA) matching networks more easy and convenient.

\section{Introduction}

Matching networks is a core problem in RF circuits design. Good impedance matching maximizes power transportation without phase shift of the voltage from the source to the load. Un-matched status of impedance could bring about a series of serious problems, like power instability, additional power loss, additional distortion and quasi-noise [1].

Usually the lumped-element matching techniques such as L-type matching networks and transmission line matching techniques using single or double stub microstrip lines are widely adapted in matching networks design [2]. By theoretical calculations or using the concept of Smith Chart to obtain the values of applied inductors and capacitors, the input impedance of transistors can be matched to the source impedance. However, the accuracy of the applied lumped-element is limited, whose values are apparently different from those obtained by theoretical analysis. Furthermore, the placed elements on PCB need microstrip lines to be placed between them as bonding pads and connecting lines. Those utilized microstrip lines however present some relatively low inductance values about $2 \mathrm{nH} 3 \mathrm{nH}$ as their length and width is rather small [3]. This extra inductance may degrade the matching network or even the whole circuit's performance, which will be discussed in the section III in detail.

The matching networks using transmission line matching techniques is more accurate than the former ones because there is no need for soldering. However, the dimension is larger as the length of many applied stubs is between $\lambda / 4$ and $\lambda / 8$, and such a matching network need add extra capacitors to be DC blocks.

This paper presents a matching network composed of transmission line and lumped elements. Compared to the traditional design, the proposed matching network has the advantages of both lumped elements and transmission lines techniques. By taking advantage of the commercial software Advanced Design System (ADS), it presents good impedance matching results and its size is more compact. Meanwhile, the applied microstrip line is not only used as a part of matching network, but also as bonding pads and connecting lines for the inductors and capacitors and it can be resized to compensate the errors brought by real elements. The table 1 shows that this design has 
good impedance matching and more compact size compared to other designs.

\section{Theoretical Analysis}

Matching Network. Inductors and capacitors connected in an L-section configuration are widely used as impedance matching circuit elements, which contains two basic lumped elements impedance matching topology, as shown in Fig 1. The impedances of the series reactance and shunt susceptance elements are represented by $\mathrm{jX}$ and $\mathrm{jB}$, respectively. Those two basic topology can match any impedance located at anywhere of the Smith Chart [1].

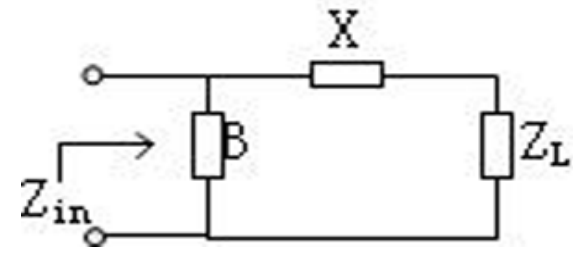

(a)

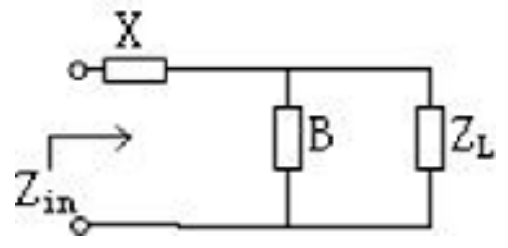

(b)

Fig 1 Basic L-type impedance matching topology

When the configuration in Fig. 1(a) is used and

$$
\begin{gathered}
Z_{\text {in }}=Z_{0}=\left[j B+\frac{1}{R_{L}+j\left(X+x_{L}\right)}\right]^{-1} \\
\mathrm{X}= \pm \sqrt{R_{L}\left(Z_{0}-R_{L}\right)}-X_{L} \\
\mathrm{~B}= \pm \frac{\sqrt{\left(Z_{0}-R_{L}\right) / R_{L}}}{z_{0}} .
\end{gathered}
$$

If the configuration in Fig 1(b) is used and

$$
\begin{gathered}
Z_{\text {in }}=Z_{0}=j X+\frac{1}{j \mathrm{~B}\left(R_{L}+j X_{L}\right)^{-1}} \\
\mathrm{~B}=\frac{x_{L} \pm \sqrt{R_{L} / z_{0}} \sqrt{R_{L}^{2}+x_{L}^{2}-z_{0} R_{L}}}{R_{L}^{2}+x_{L}^{2}} \\
\mathrm{X}=\frac{1}{B}+\frac{x_{L} Z_{0}}{R_{L}}-\frac{z_{0}}{B R_{L}} .
\end{gathered}
$$

Short Transmission Line Theory. Microstrip lines are added between the lumped elements in the proposed circuit as bonding pads and connecting lines and those microstrip lines are treated as short transmission lines sections.

The impedance parameters of a length $l$ of line having characteristic impedance are as follow 


$$
\begin{aligned}
& Z_{12}=Z_{21}=-j Z_{0} \csc \beta l \\
& Z_{11}=Z_{22}=-j Z_{0} \cot \beta l
\end{aligned}
$$

The series elements of the T-equivalent circuit are

$$
Z_{11}-Z_{12}=-j Z_{0} \tan \left(\frac{\beta l}{2}\right)
$$

while the shunt element of the T-equivalent is .

$$
\begin{aligned}
& \frac{\pi}{2}=Z_{0} \tan \left(\frac{\beta l}{2}\right) \\
& \mathrm{B}=\frac{1}{z_{0}} \sin \beta l
\end{aligned}
$$

For a short length of line with large characteristic impedance, then (10) and (11) approximately reduce to

$$
\begin{gathered}
\mathrm{x} \approx Z_{0} \beta L \\
\mathrm{~B} \approx 0
\end{gathered}
$$

The footprint 0402 or 0603 dimensions are widely used in capacitors and inductors. In order to match the footprint of those applied lumped elements for eliminating the effect caused by discontinuities, therefore, the width of those selected microstrip lines is very small. According to the theory above, the utilized microstrip lines are equivalent to inductors.

The Proposed Matching Network. As mentioned in (13) and (14), a short and narrow microstrip placed between lumped elements as bonding pads and connecting lines as shown in Fig. 2 , are able to be treated as inductors. The microstrip lines and lumped elements are regarded as a whole reactive element so long as the whole series inductance and the shunt susceptance are satisfied to (2), (3), (5) and (6).

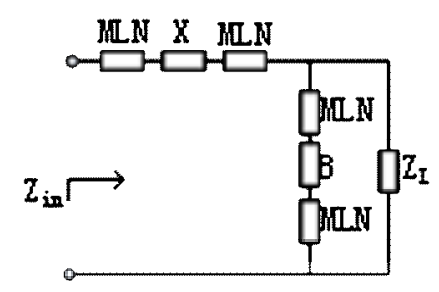

Fig 2 The proposed matching network topology

As shown in Fig 3

$$
\left\{\begin{array}{c}
2 \mathrm{j} \omega \mathrm{L}+\mathrm{jx^{ \prime }}=\mathrm{jx} \\
\frac{2}{j \omega L}+\mathrm{jB}^{\prime}=\mathrm{jB}
\end{array}\right.
$$




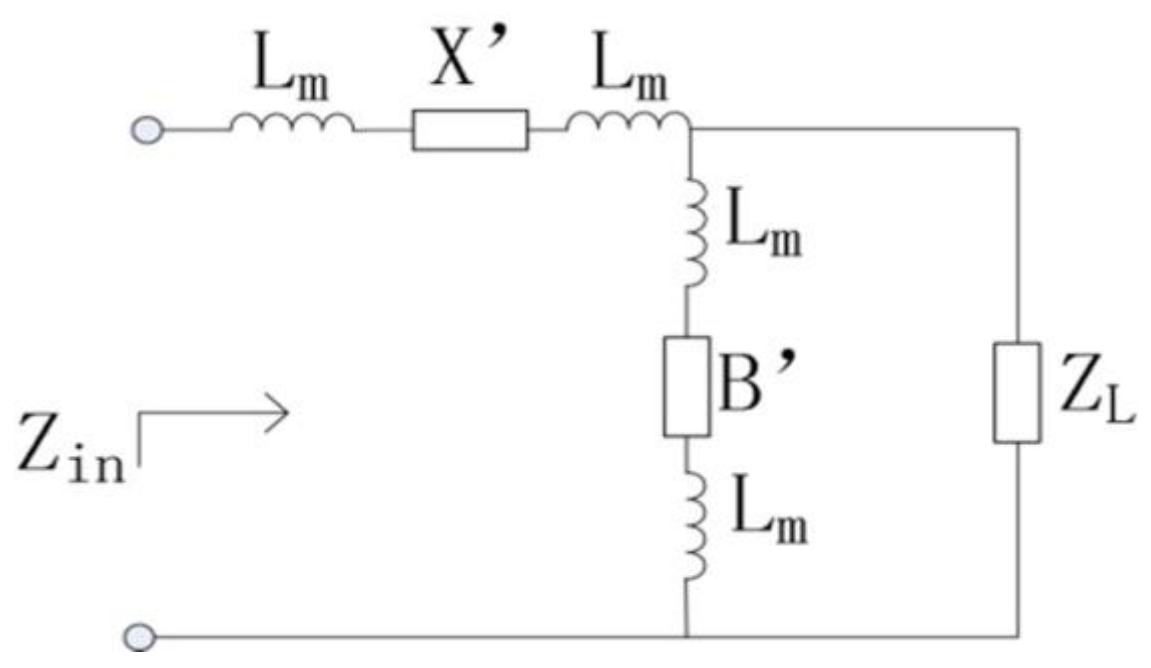

Fig. 3 Schematic of the proposed matching network

\section{Example}

A matching example is presented for a LNA in our R\&D program operating at $1.2 \mathrm{GHZ}-1.25$ GHZ for the proposed matching network. The type of the applied pHEMT is ATF-54143 provided by Agilent and the bias voltage and current is $3 \mathrm{~V}$ and $38 \mathrm{~mA}$ respectively.

The calculation of the inductance presented by microstrip lines is very intricate, so the simulation software ADS is implemented. The pHEMT is matched by ideal lumped elements as shown in Fig 4.

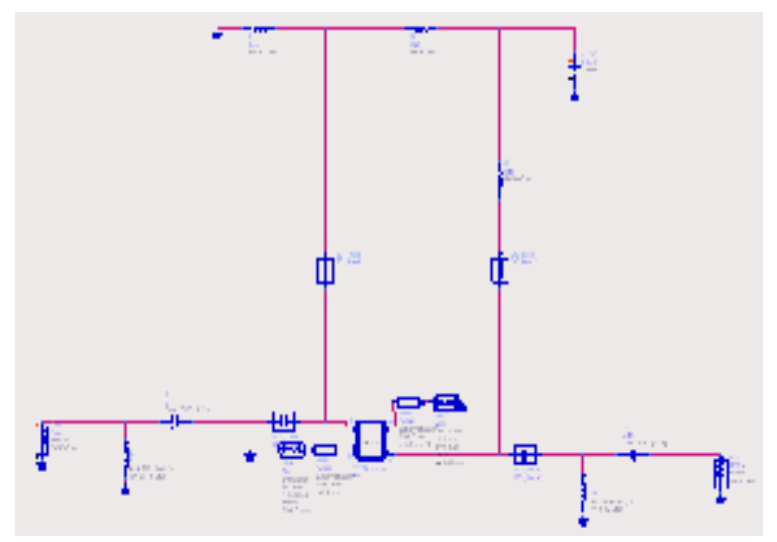

Fig 4 Schematic of the LNA

Considering simultaneous approach of both $\mathrm{NF}_{\min }$ and $\mathrm{G}_{\max }$, the $\mathrm{S} 11$ is slightly larger than the S22. It can be seen from the simulation results presented in Fig 5 The input and output ports are matched well when the ideal lumped elements are applied. 


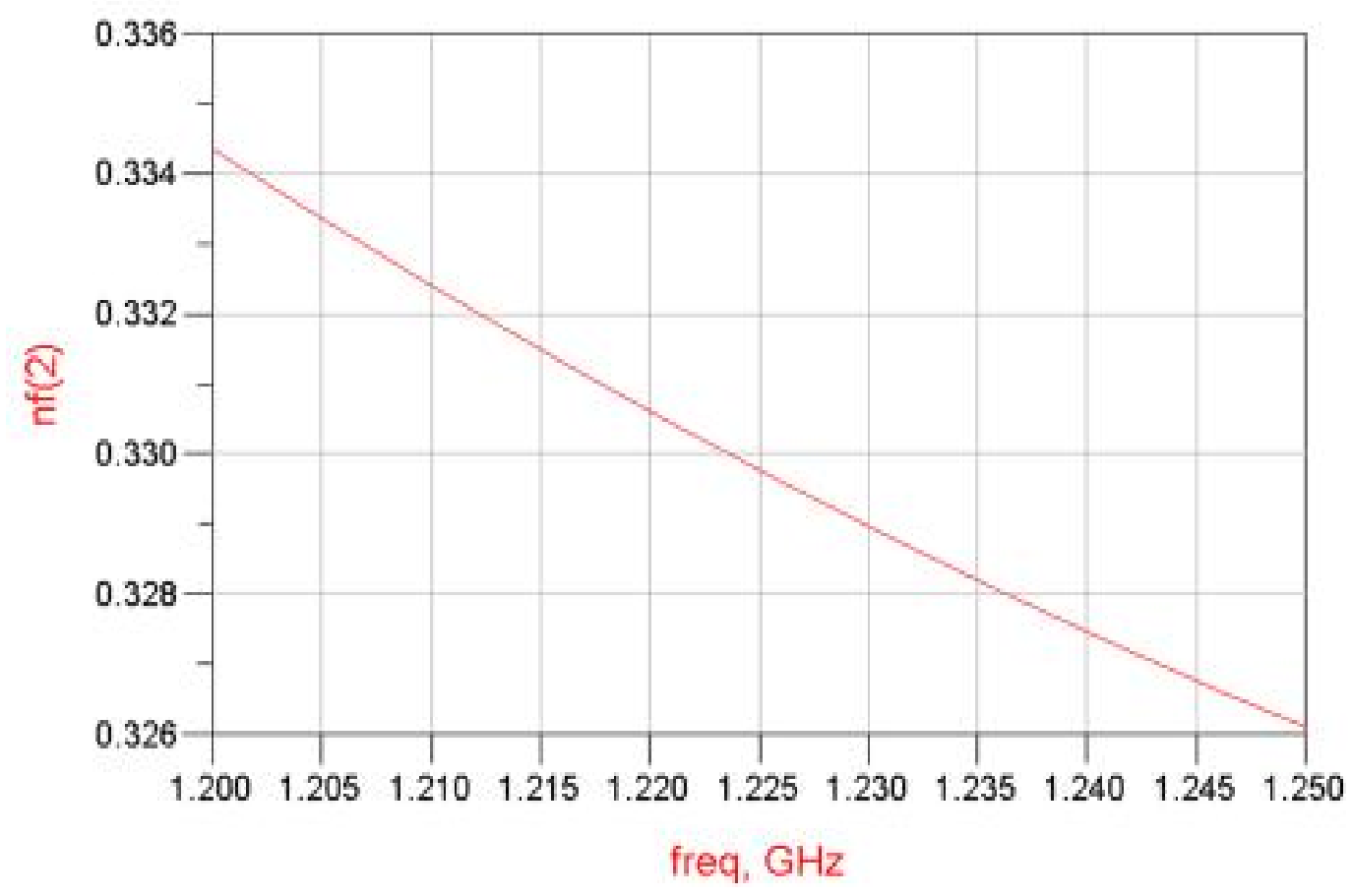

(a) NF

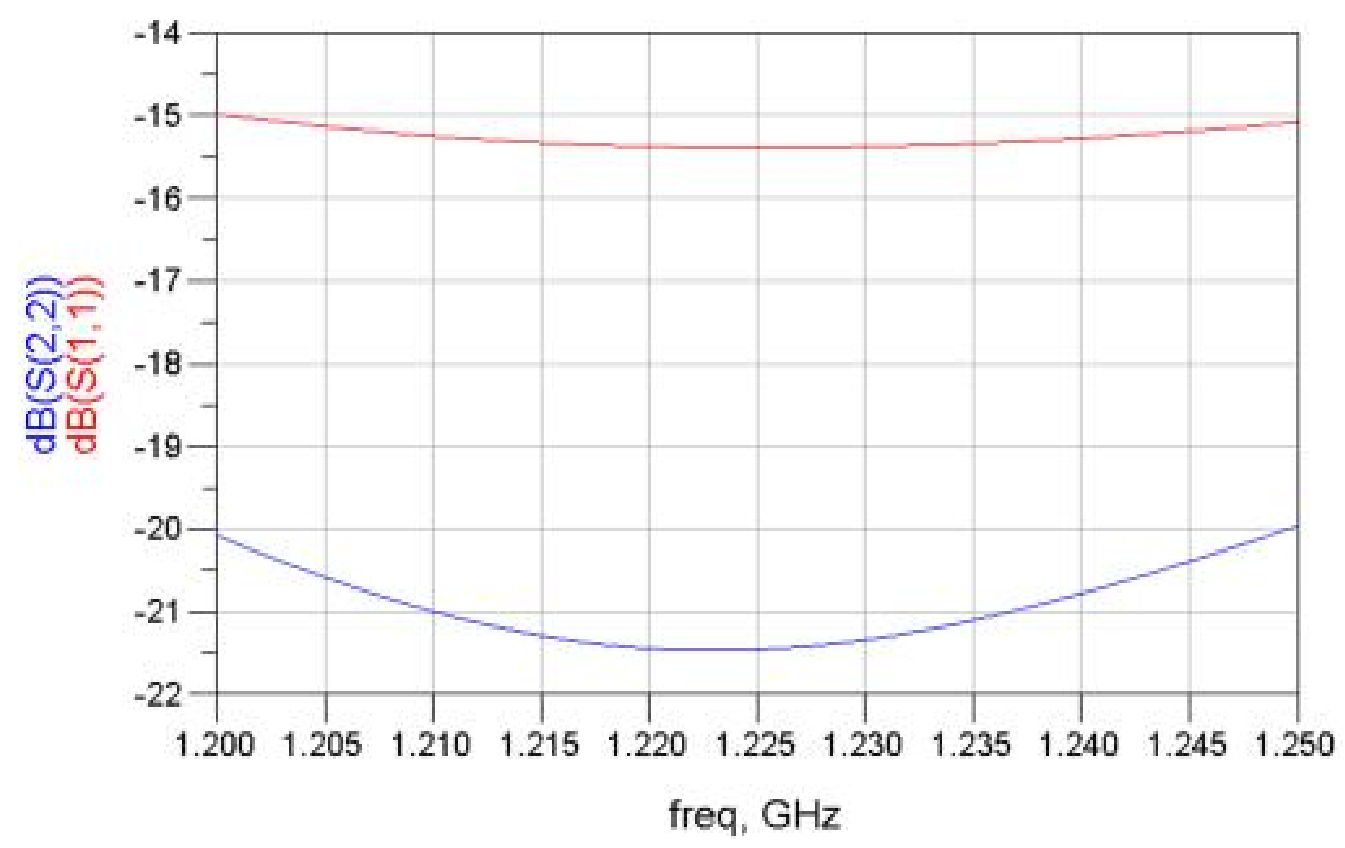

(b) Return Loss

Fig 5 The NF and return loss in LNA applied ideal elements

After microstrip lines are placed between lumped elements as bonding pads and connecting lines, the both of input and output matching networks are severely degraded as depicted in Fig. 6 This is because of the inductance caused by the placed microstrip lines, which is cannot be ignored.

Choosing the optimizers in ADS shown in Fig6, it can compensate the errors brought by extra placed microstrip lines by adjusting the values of those inductors and capacitors. When the optimization is done, the results are shown in Fig 8. 


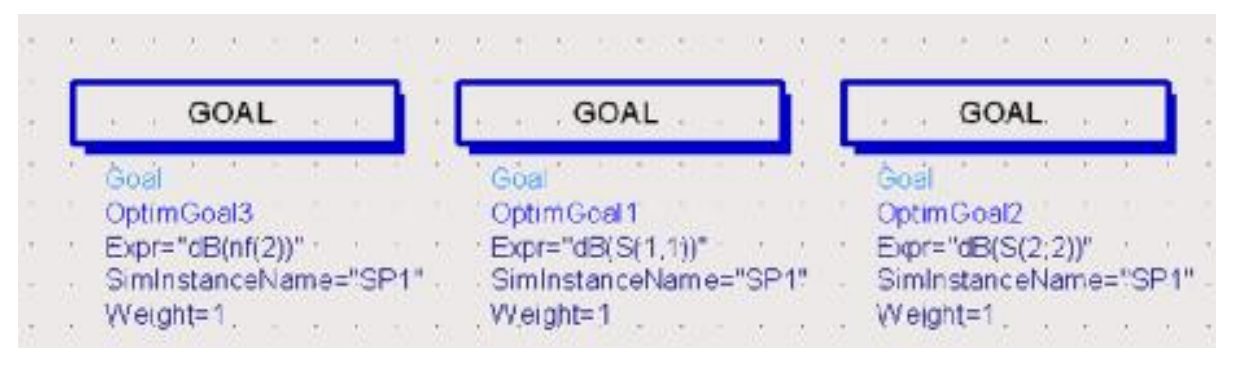

Fig 6 Optimizers in ADS

It can be seen from the above, the established matching network is composed of three elements, inductors, capacitors and microstrip lines, which all played a role in impedance matching.

Finally, when substituting real inductors and capacitors for the ideal ones, there are inevitable errors causing poor performance in matching network, as shown in Fig 7.

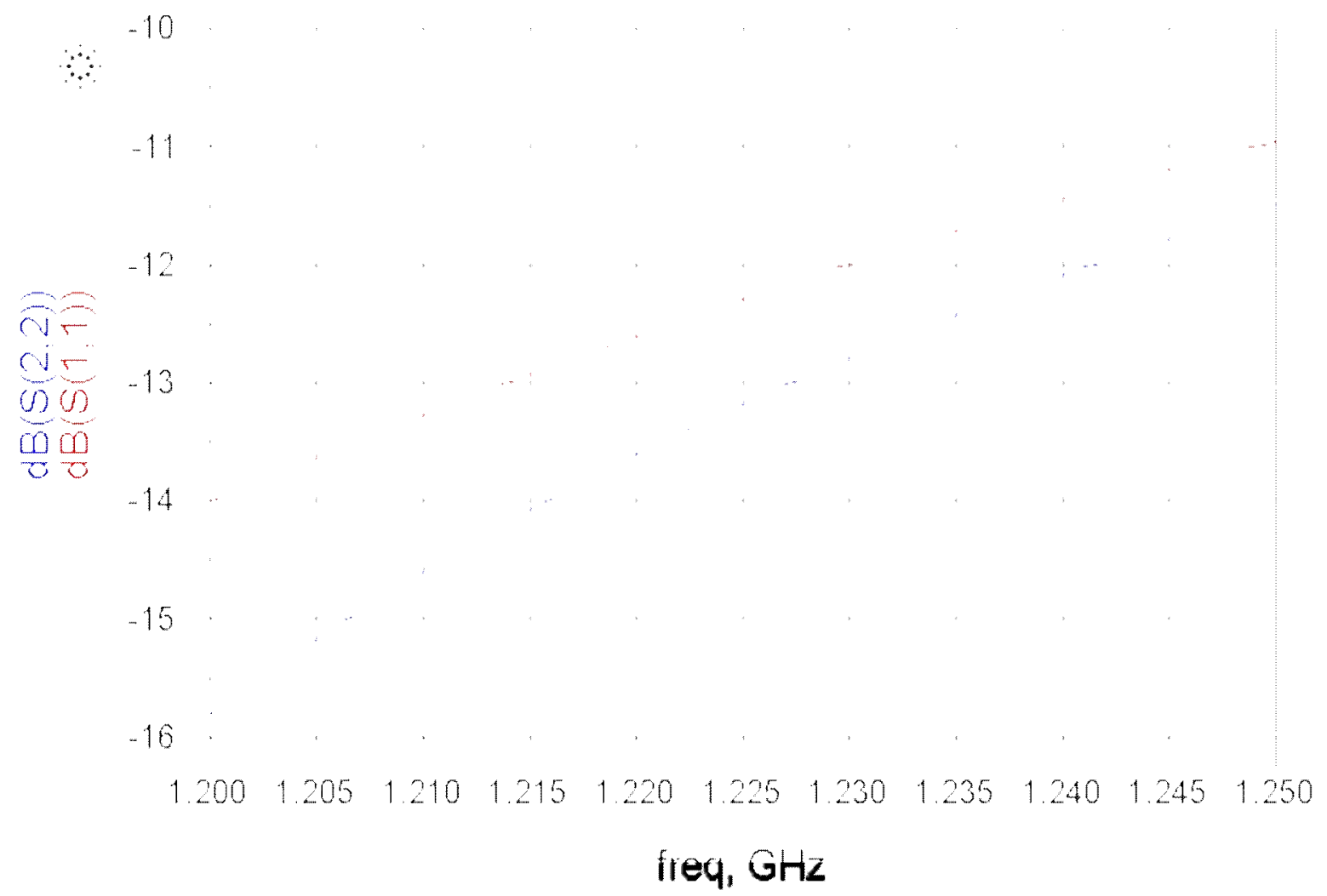

Fig 7 Simulation results in LNA applied real elements

By adjusting the length of microstrip lines, the error can be compensated without replacing any new lumped elements, as shown in Fig 8. 


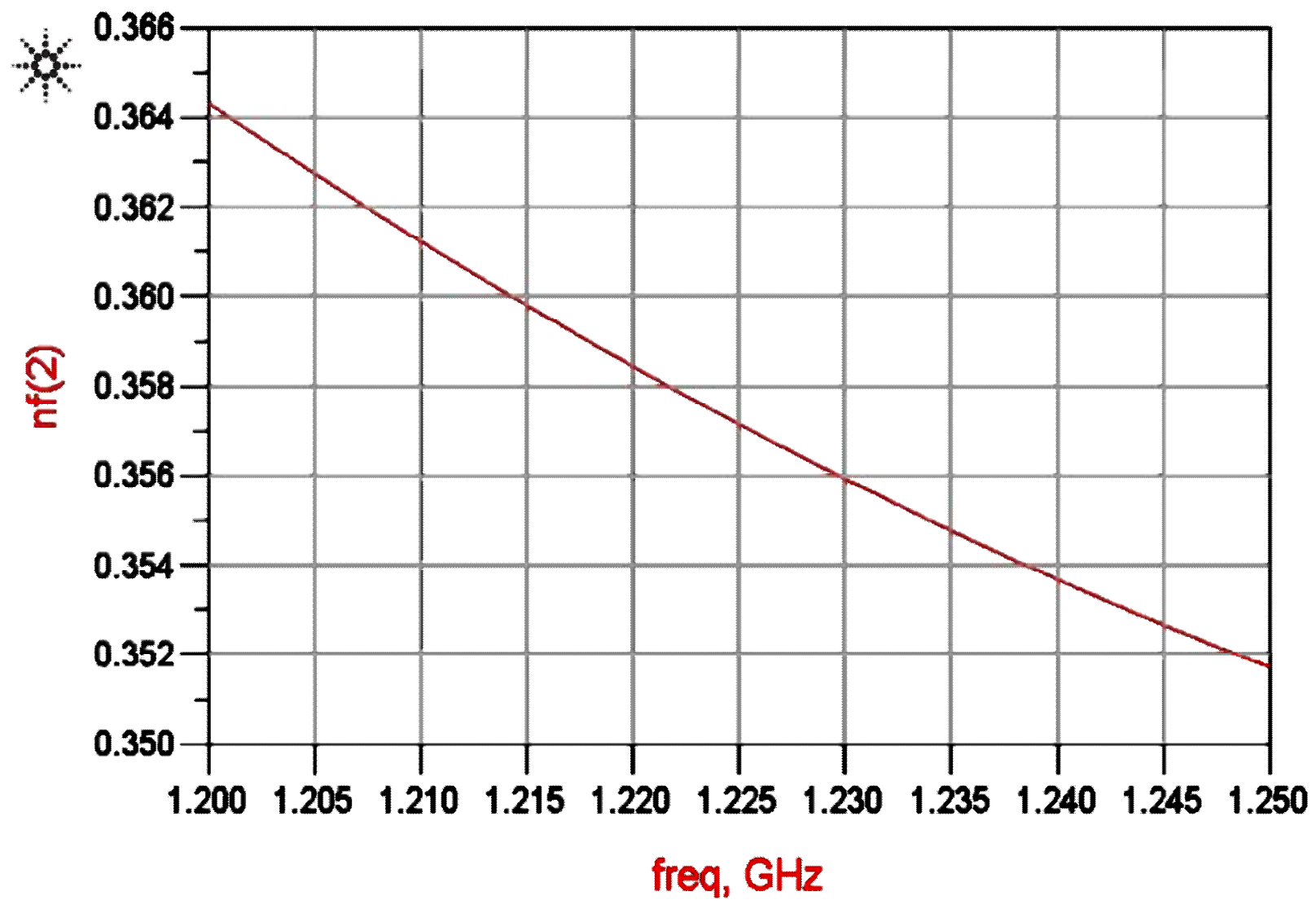

(a)NF 
In layout shown in Fig 9, the microstrip lines are used as bonding pads and connecting lines. The dimension of the whole circuit is only $16 \mathrm{~mm}$.

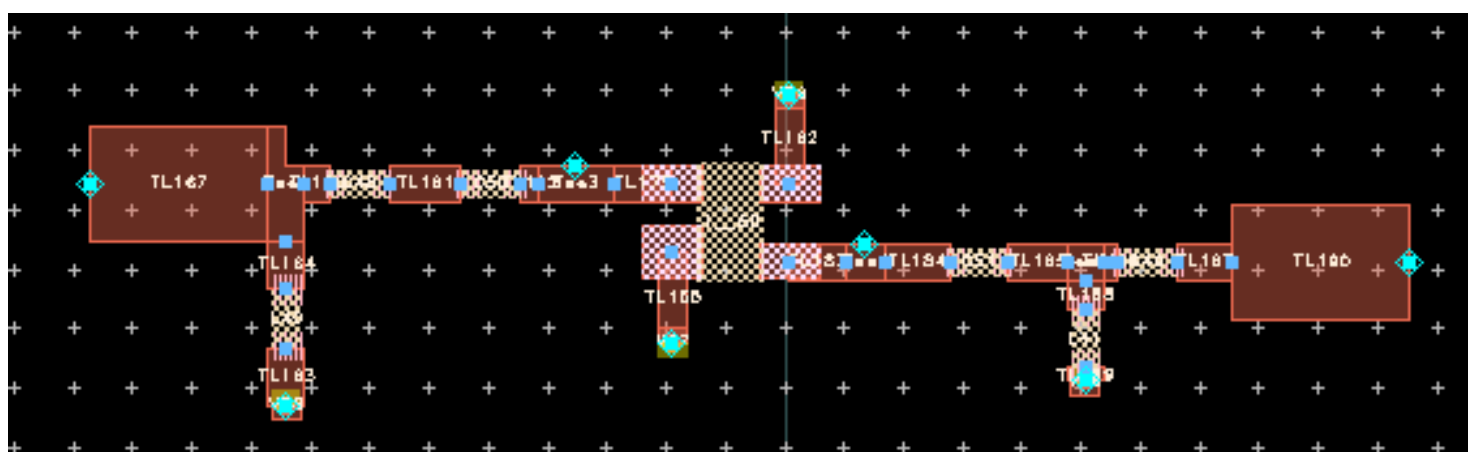

Fig 9 The layout of the matching netwolk

\section{Conclusion}

The design of a practical matching network is a core problem in RF circuit. In this respect, the CAD tools are utilized. These packages are useful to optimize the circuit performance by working on the elements values.

In this proposed method, the microstrip lines as short transmission lines are analyzed. Theoretical analysis showed that the microstrip lines can be not only used as bonding pads and connecting lines between the lumped elements on PCB, but also a part of matching networks. Simulation results presented that the proposed matching network is more compact and accurate because the resizable microstrip lines are utilized. The Table 1. shows that this design has good impedance matching and more compact size compared to other designs. Such a matching network has some reference for LNA and even other RF circuits.

Table 1. Comparison of different design

\begin{tabular}{|c|c|c|c|c|}
\hline \multirow[t]{2}{*}{ Ref } & \multicolumn{4}{|c|}{ Comparison of different disign } \\
\hline & $\begin{array}{l}\text { Matching } \\
\text { Technique }\end{array}$ & $\mathrm{NF}[\mathrm{dB}]$ & $\mathrm{S} 11[\mathrm{~dB}]$ & $\mathrm{S} 22[\mathrm{~dB}]$ \\
\hline$[5]$ & Transmission line & 0.977 & -9.2 & -17 \\
\hline$[6]$ & Transmission line & $<3.5$ & - & - \\
\hline [7] & Transmission line & 2.463 & -15.22 & -16.47 \\
\hline [8] & Transmission line & 0.522 & $>-10$ & $>-68$ \\
\hline [9] & Lumped-element & 0.586 & -15 & -30 \\
\hline$[10]$ & Lumped-element & 0.451 & -14.023 & -8.379 \\
\hline $\begin{array}{l}\text { This } \\
\text { work }\end{array}$ & $\begin{array}{l}\text { Integrated with } \\
\text { both }\end{array}$ & 0.35 & -20 & -20 \\
\hline
\end{tabular}




\section{Acknowledgement}

The work was financially supported by Guangzhou Science Technology and Innovation Commission (Grant Nos. 201510010093).

\section{Reference}

[1] Richard Cchi-Hsi Li: Key Issues in RF/RFIC Circuit Design : (Higher Education Press, 2005).

[2] I.Bahl, Fundamentals of RF and Microwave Transistor Amplifiers, (John Wiley \& Sons, 2009)

[3] David M. Microwave engineering. (John Wiley \& Sons, 2012).

[4] Yarman, B. S., M. Sengul, and A. Kilinc: Design of Practical Matching Networks with Lumped Elements via Modeling. Circuits \& Systems I Regular Papers IEEE Transactions on 54.8(2007):1829-1837.

[5] Giri, Santu Kr, and Chayanika Bose: Design and Development of An S-band Low Noise Amplifier. 2010 Annual IEEE India Conference (INDICON). IEEE, 2010.

[6] Lianjuan, Tao, and Bao Jingfu. An 0.5-6GHz Ultra-Wideband Low Noise Amplifier Design. Electronics, Communications and Control (ICECC), 2011 International Conference on. IEEE, 2011.

[7] Rizk, M., M. Nasar, and A. Hafez: Design and Simulation of High Gain PHEMT Low Noise Amplifier (LNA). International Conference on Computer Engineering \& Systems 2007:253 - 257.

[8] Khan, Muhammad Saad, et al. PHEMT Based Wideband LNA for Wireless Applications. China Communications 12.10 (2015): 108-116.

[9] Meng, Qing Bin, et al: Design and Simulation for Multi-stage Matching Networks of a Low-noise Amplifier. Journal of Microwaves (2012).

[10]Zhi-Xi, Y. U., et al: Design and Simulation of BeiDou Navigation Receiver LNA. Modern Electronics Technique (2012). 